\title{
A NEW LINEAR DIFFUSION METHOD FOR THE MICROBIOLOGICAL ASSAY OF STREPTOMYCIN AND DIHYDROSTREPTOMYCIN ${ }^{1}$
}

\author{
By J. M. McGUIRE, W. W. DAVIS, T. V. PARKE, and W. A. DAILY
}

(From the Lilly Research Laboratories, Indianapolis, Ind.)

A new antibiotic assay method has been developed which involves a radically different diffusion system from that employed in plate methods. In this new method diffusion occurs from a solution of the antibiotic in a test tube into a column of agar contained in a glass capillary.

This new method promises improved accuracy because the linear diffusion system has important advantages over the diffusion system presented in the plate methods. Secondly, this method provides greater efficiency in handling test equipment than is possible for other methods. Capillaries can be filled, read, and cleaned by semiautomatic procedures. Test tubes and other essential equipment are efficiently cleaned in a special steam cabinet. Finally, the method affords comparative economy in personnel and materials over other methods.

The method is inherently capable of extension to testing of other antibiotic or germicidal materials, but has been worked out in detail and applied only for the testing of streptomycin and dihydrostreptomycin. The obvious extension to testing of penicillin is being studied.

The test depends upon the formation of characteristic zones in the seeded agar column in the capillary. Figure 1 shows a section of a capillary in which such zones have formed.

A sharp initial boundary for diffusion of antibiotic from solution into the agar column is provided by the molded end of the agar column. A region of inhibition of growth occurs at this end of the agar column. The length of this region depends on concentration of antibiotic in the test solution. This region is bounded by a sharp dense zone of aerobic growth of the test organisms which allows the length of this region to be accurately measured. This sharp zone of aerobic growth results from the fact that oxygen diffuses unused

\footnotetext{
1 Presented at the Second National Symposium on Recent Advances in Antibiotics Research held in Washington, D. C., April 11-12, 1949, under the auspices of the Antibiotics Study Section, National Institutes of Health, Public Health Service, Federal Security Agency.
}

through the region of inhibition and becomes available to the first uninhibited organisms encountered. Since this growth uses all the oxygen available by diffusion inward, the growth of organisms beyond this zone is limited by the oxygen originally dissolved in the agar. Accordingly, growth in this region is very light. A pellicle of dense aerobic growth is always seen at the top surface of the agar column.

Figure 2 shows the manner in which the length of the inhibition region depends upon the concentration of dihydrostreptomycin in test solutions. Such a standard curve is prepared each day from known dilutions of a standard solution. The results on unknown solutions are referred to this curve for evaluation.

\section{METHOD}

A complete description of the required equipment and the details of the method is being published elsewhere. Only a brief description of the operations involved in the use of this method can be given here.

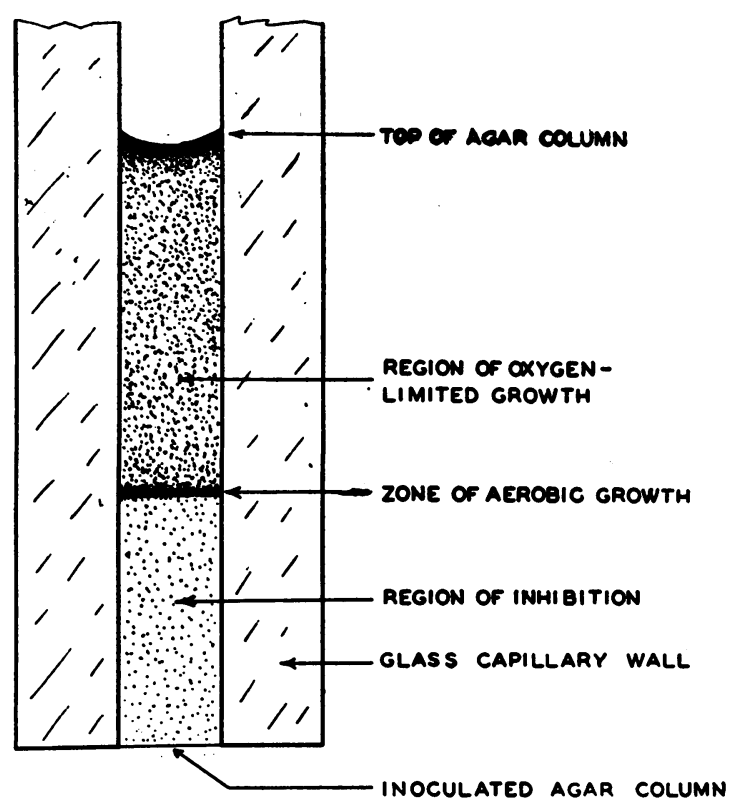

Fig. 1. Schematic Drawing Showing the Regions of Growth in the Agar Colum 


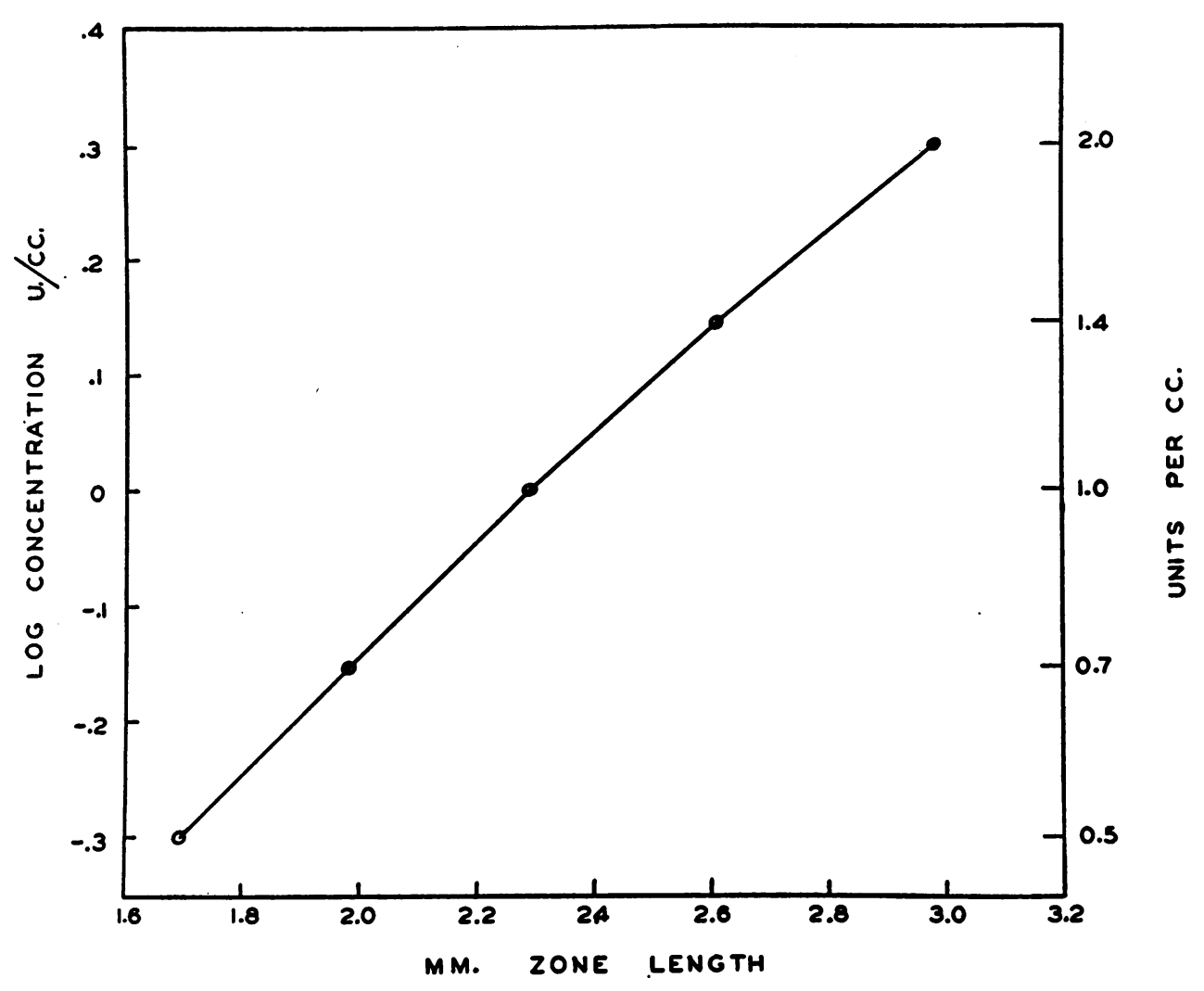

Fig. 2. A Typical Curve Showing the Dependence of Zone Length on Concentration of Antibiotic

The capillaries employed are constructed of pyrex capillary tubing of approximately $1.5 \mathrm{~mm}$. bore and approximately $7 \mathrm{~mm}$. outside diameter. They are cut to approximately $3 \frac{1}{2}$ inches. One end is ground flat while the other is only edge ground.

Clean dry capillaries are partially filled by placing them in an automatic filling device containing plain $4 \%$ melted agar seeded with spores of Bacillus subtilis. This device fills 800 capillaries simultaneously. After the capillaries are filled, the entire assembly is cooled to allow solidification of the agar. This operation provides a flat molded end on the agar column in each capillary.

After cooling, the filled capillaries are removed from the filling device and placed two each in test tubes containing roughly $2 \mathrm{ml}$. of test solution. The test solutions have been made up by diluting standard and unknown antibiotic solutions with nutrient broth. The concentrations of standard test solutions are from 0.5 to 2.0 units per $\mathrm{ml}$, while the unknowns are diluted to bring them tc approximately 1 unit per $\mathrm{ml}$. The test tubes are capped and incubated in racks in a constant temperature water bath at $32^{\circ} \mathrm{C}$. overnight. After incubation the contents of a group of test tubes (usually five) containing 'a single test solution are dumped on a draining device. The capillaries are rinsed with water and then dried on an absorbent surface.
The capillaries are then transferred in a group to a special carriage provided on a modified Fisher-Lilly Antibiotic Zone Reader. The modification of the reader permits the length of the zone of inhibition to be read reproducibly to plus or minus $0.01 \mathrm{~mm}$.

After the reading, the capillaries are stacked in a wire basket and cleaned simultaneously in the steam cleaning cabinet. Test tubes and bottles used for dilutions are cleaned in a similar manner.

The filling device formerly employed allowed simultaneous filling of 400 capillaries. Three units of test equipment are now being constructed, each designed to fill and handle 800 capillaries per day. Each unit anticipates the performance of 57 six-tube tests or 114 threetube tests in addition to a complete standard curve.

\section{RESULTS AND CONCLUSIONS}

Deviations of test results on a 1 unit per $\mathrm{ml}$. solution have been studied employing the discplate method and the capillary-linear diffusion method. Fifty Petri dishes were employed in each plate-disc experiment and 50 test tubes in each capillary experiment. The standard deviations for six plate-disc experiments were from 
$7 \%$ to $10 \%$ for individual zones, and those for seven capillary experiments were from $3 \%$ to $8 \%$ for individual capillaries. A similar experiment was performed to study the deviations of the broth-turbidimetric assay method, employing 150 tubes. The standard deviation of this method was found to be $3 \%$. These data indicate that when the two diffusion assay methods are compared on the basis of equivalent numbers of plates and pairs of capillaries, the linear diffusion capillary method is somewhat superior to the plate method in precision. In comparison to the brothturbidimetric assay method the capillary method is less precise but more convenient. 